\title{
Lobar and sublobar resection with and without brachytherapy for small stage IA non-small cell lung cancer
}

\author{
Hiran C. Fernando, FRCS, FRCSEd ${ }^{\mathrm{a}}$ \\ Ricardo S. Santos, MDa \\ John R Benfield, MD ${ }^{\mathrm{c}}$ \\ Frederic W. Grannis, MD ${ }^{d}$ \\ Robert J. Keenan, MD \\ James D. Luketich, MD ${ }^{a}$ \\ John M. Close, MA, PMSD ${ }^{b}$ \\ Rodney J. Landreneau, MD ${ }^{\mathrm{a}}$
}

From the Division of Thoracic and Foregut Surgery and Department of Dental Public Health $^{\mathrm{b}}$, University of Pittsburgh Medical Center, Pittsburgh, Pa, the Division of Thoracic Surgery, David Geffen School of Medicine at UCLA, Los Angeles, Calif ${ }^{c}$, the Division of Thoracic Surgery, City of Hope Medical Center, Duarte, Calif ${ }^{\mathrm{d}}$, and the Division of Thoracic Surgery, Allegheny General Hospital, Pittsburgh, $\mathrm{Pa}^{\mathrm{e}}$

Read at the Eighty-fourth Annual Meeting of The American Association for Thoracic Surgery, Toronto, Ontario, Canada, April 25-28, 2004.

Received for publication April 22, 2004; revisions received Aug 26, 2004; accepted for publication Sept 3, 2004.

Address for reprints: Hiran C. Fernando, FRCS, Associate Professor, Department of Cardiothoracic Surgery, Boston Medical Center, 88 East Newton St, Robinson B402, Boston, MA 02118 (E-mail: hiran. fernando@bmc.org).

J Thorac Cardiovasc Surg 2005;129:261-7

$0022-5223 / \$ 30.00$

Copyright $\odot 2005$ by The American Association for Thoracic Surgery

doi:10.1016/j.jtcvs.2004.09.025

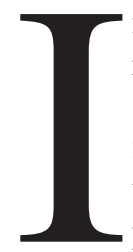

n 1995, the Lung Cancer Study Group (LCSG) reported the first and only randomized study to compare sublobar resection (SR) and lobar resection (LR) for T1 N0 non-small cell lung cancer (NSCLC). ${ }^{1}$ The principal finding in this was a 3-fold increase in local recurrence among patients who underwent SR. Two years later, a multicenter nonrandomized study compared patients who underwent SR (open or video-assisted thoracoscopic wedge resection) to lobectomy and demonstrated a trend toward increased local recurrence in the patients who did so. ${ }^{2}$ As a result of these and other studies, LR has remained the preferred treatment for NSCLC at most centers. 
TABLE 1. Recurrence rates for tumors smaller than $2 \mathrm{~cm}$

\begin{tabular}{lrcc}
\hline & LR $(\mathbf{n}=\mathbf{8 0})$ & SR $(\mathbf{n}=57)^{*}$ & $\boldsymbol{P}$ value \\
\hline Local recurrence & $8(10.0 \%)$ & $10(17.5 \%)$ & .20 \\
Distal recurrence & $15(18.8 \%)$ & $8(14 \%)$ & .47 \\
\hline
\end{tabular}

*Brachytherapy was used in 22 SRs $(38.6 \%)$.

A number of factors are now leading us to reevaluate the optimal treatment for NSCLC. First is an issue of size. Larger tumors are more likely to display regional or distant metastases at the time of diagnosis. A Japanese study compared behavior of TI N0 tumors smaller and larger than 2 $\mathrm{cm} .{ }^{3}$ Patients with the larger tumors had an overall poorer prognosis and an increased incidence of lymphatic and hematogenous metastases. During the past few years, the increasing prevalence of computed tomographic scan screening programs has led to the detection of ever smaller $\mathrm{NSCLCs}^{4}$; these small tumors may be less aggressive than larger stage I neoplasms. Therefore, removal of a relatively large amount of normal lung by LR might be unnecessary and at times potentially harmful. The use of SR for these smaller tumors, even for normal-risk patients, is increasingly supported by reports that are emerging, particularly from Asia. ${ }^{5,6}$

In light of these reports and concerns regarding the optimal resection for small NSCLC, we conducted a multicenter retrospective review to compare outcomes of LR with SR for patients with T1 N0 cancers. Specific emphasis in this North American experience has been placed on the impact of tumor size on outcome. In addition, the more recent operations have included the use of adjuvant intraoperative brachytherapy. The effectiveness of this technique in improving local control was also evaluated.

\section{Patients and Methods}

Patients were selected from the research databases of four centers. Institutional review board approval was obtained, and patient identifiers were removed from the pooled data in the common database. Only patients with pathologic T1 N0 NSCLC who underwent SR or LR were included. There were no pneumonectomies in this series.

Patients were selected for SR if they were considered to be at increased risk with LR. SR procedures included segmental or wedge resections with pathologically confirmed clear surgical margins. All patients underwent either lymph node sampling or lymph node dissection. The approach used (video-assisted thoracic surgery versus open procedure) was not recorded in the common data set.

In an effort to decrease local recurrence, adjuvant intraoperative brachytherapy was used in several of the more recent SR cases. ${ }^{7}$ In these cases, an iodine 125 implant was fashioned and placed on the staple line. The ${ }^{125}$ I seeds were embedded in a Vicryl polyglactin suture (Ethicon). These sutures were ordered in advance from the manufacturer (Amersham Health, Princeton, NJ).
The ${ }^{125}$ I sutures were sewn onto a piece of polyglyconate mesh (Ethicon) at an appropriate spacing to achieve a prescribed dose of 10,000 to $12,000 \mathrm{cGy}$ to a $0.5-\mathrm{cm}$ depth. The ${ }^{125} \mathrm{I}$ implant was then secured over the staple line by the thoracic surgeon with an approximate $2-\mathrm{cm}$ margin. The mesh was secured to the lung with interrupted 3-0 silk sutures.

Patients were divided into two groups. The first consisted of those with tumors smaller than $2 \mathrm{~cm}$. The second group included patients with tumors measuring 2 to $3 \mathrm{~cm}$. In each group, an analysis of SR versus LR was performed. In particular, the incidences of local and distal recurrence and survival were recorded. Local recurrence was defined as any recurrence within the same lobe of the lung, or interlobar and hilar lymph nodes (N1 nodes). All other metastases were classified as distant recurrence. If a patient had both local and distal recurrence, they were recorded separately to get the incidence of each.

After removal of patient identifying information, data from the study sites was combined and entered into an SPSS file for Windows (version 11.0; SPSS Inc, Chicago, Ill). Statistical analysis included $t$ test analysis of quantitative data, $\chi^{2}$ analysis of dichotomous data, and Kaplan-Meier survival analysis with log-rank analysis to compare groups.

\section{Results}

There were 291 patients with T1 N0 disease, including 145 men and 146 women, in this study. The resections performed included $167 \mathrm{LRs}$ and 124 SRs. There were 73 (58.9\%) segmentectomies and 51 (41.1\%) wedge resections in the SR group. The SR group patients were older $(68.4 \pm$ 8 years) than the LR group patients $(66.1 \pm 7.8$ years; $P=$ $.018)$. Additionally, pulmonary function was significantly worse $(P=.001)$ in the SR group (forced expiratory volume in 1 second $\left[\mathrm{FEV}_{1}\right] 53.1 \% \pm 21.3 \%$ ) than in the LR group $(78.2 \% \pm 22.5 \%)$. Patients were stratified into those with tumors smaller than $2 \mathrm{~cm}$ maximum diameter $(\mathrm{n}=137)$ and those with tumors ranging from 2 to $3 \mathrm{~cm}(\mathrm{n}=154)$. The histologic types of the cancers were as follows: adenocarcinoma $(\mathrm{n}=127)$, squamous $(\mathrm{n}=117)$ large cell or adenosquamous $(n=27)$, and bronchiolar $(n=20)$. There was no preponderance of bronchiolar cancers in either group, with only $12(7.2 \%)$ in the LR group and $8(6.5 \%)$ in the SR group having bronchiolar tumors. Since 1997, adjuvant brachytherapy has been used in conjunction with SR with increasing frequency in two of the study centers. Among the 124 SR resections, brachytherapy was performed in 60 cases $(48 \%)$, with greater use $(P=.05)$ for the 2 - to 3 -cm tumors $(\mathrm{n}=38,55.9 \%)$ than for those smaller than $2 \mathrm{~cm}(\mathrm{n}=22,38.6 \%)$. Brachytherapy was used in similar $(P=.46)$ proportions of the wedge $(45 \%)$ and segmental $(52 \%)$ resections. The mean follow-up for all patients was 34.5 months.

\section{Tumor Diameter Less Than $2 \mathrm{~cm}$}

Recurrence rates for the group with tumors smaller than 2 $\mathrm{cm}$ are illustrated in Table 1 . As with many other series, 
TABLE 2. Recurrence rates for 2- to 3-cm tumors

\begin{tabular}{lccc}
\hline & LR $(\mathbf{n}=\mathbf{8 6})$ & SR $(\mathbf{n}=\mathbf{6 8})^{*}$ & $\boldsymbol{P}$ value \\
\hline Local recurrence & $3(3.5 \%)$ & $3(4.4 \%)$ & .76 \\
Distal recurrence & $16(18.6 \%)$ & $20(29.4 \%)$ & .116 \\
\hline
\end{tabular}

*Brachytherapy was used in 38 SRs $(55.9 \%)$.

local recurrence had an almost 2-fold increase in the SR group. However, this difference was not statistically significant. Distal recurrence was similar between the groups. Survival is demonstrated in Figure 1). The mean and median survivals were 95.8 and 85 months after LR and 82.2 and 55.8 months after SR. These were not significantly different $(P=.97)$. Cancer caused death in 9 patients $(11.3 \%)$ in the LR group and $7(12.3 \%)$ in the SR group. These rates were not significantly $(P=.853)$ different.

\section{Tumor Diameter From 2 to $3 \mathrm{~cm}$}

Local and distant recurrence rates for the 2- to 3-cm tumors are illustrated in Table 2. The local recurrence rates were similar in the SR and LR groups. This may have been related to the greater use of adjuvant brachytherapy for the larger tumors. Survival is demonstrated in Figure 2). Mean and median survivals were 70 and 68.7 months after LR and 44.7 and 50.6 months after SR. These were significantly $(P=.003)$ different. Cancer caused death in 12 patients $(13.8 \%)$ in the LR group and $16(23.9 \%)$ in the SR group. These rates were not significantly different $(P=.108)$.

\section{Impact of Type of SR and Use of Brachytherapy on Local Recurrence}

A further subanalysis was performed of all patients undergoing SR to determine the impacts of the subtype of resection (wedge or segmentectomy) and the use of adjuvant brachytherapy on local recurrence. In the 124 patients undergoing SR, local recurrence was seen after 7 (9.6\%) of the segmentectomies and $6(11.8 \%)$ of the wedge resections. These rates were not significantly $(P=.725)$ different. On the other hand, the use of brachytherapy significantly $(P=$ .012) decreased the incidence of local recurrence, from 11 patients $(17.2 \%)$ to $2(3.3 \%)$.

\section{Discussion}

Patients with stage IA NSCLC should ideally be treated by resection. External beam radiation is traditionally used for patients who are unable to tolerate any type of pulmonary resection. However, outcomes of such radiotherapy are inferior to those of operation. In a previous study of 71 node-negative patients who received at least $60 \mathrm{~Gy}$ to their cancers, ${ }^{8} 3$ - and 5-year survivals were $19 \%$ and $12 \%$. A more recent report described results after radiation therapy in 60 patients with stage I or II cancers. ${ }^{9}$ Local progression occurred in $53 \%$ of patients, with a median progression-free

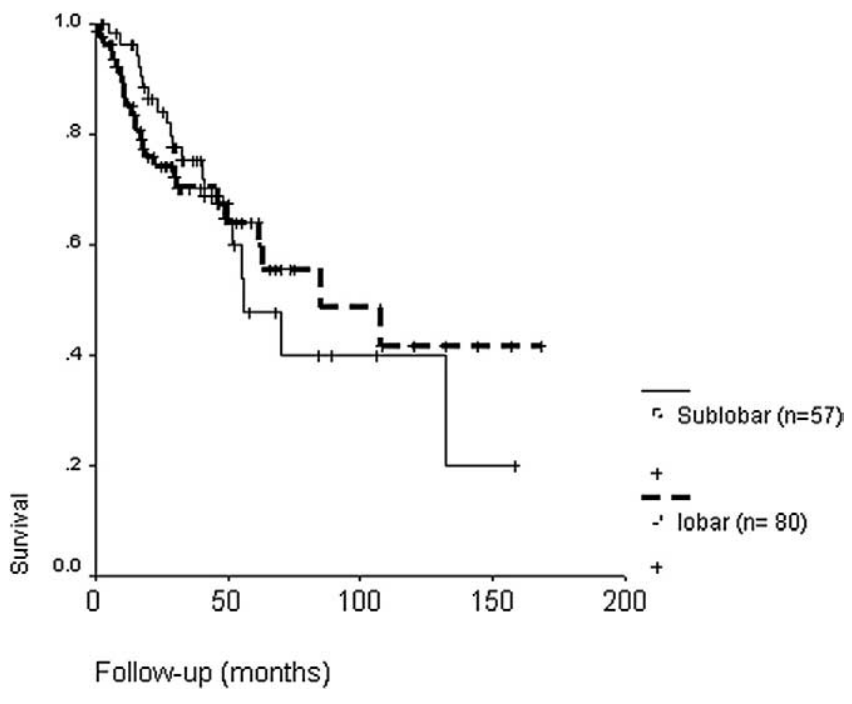

Figure 1. Survival for tumors smaller than $2 \mathrm{~cm}$; SR versus LR $(P=.97)$.

survival of 18.5 months and an overall median survival of 20 months. Another study from Duke of 141 patients with stage I disease demonstrated 2- and 5-year survivals of 39\% and $13 \%$, respectively. ${ }^{10}$ The overall median survival was 18 months. The median survival, even after SR, was much longer in our series. A new modality that may offer some advantages relative to radiation therapy is radiofrequency ablation. This modality appears to be effective, particularly for small tumors. ${ }^{11}$ Currently, however no long-term follow-up data exist. Until long-term outcomes are available, radiofrequency ablation should be reserved for patients who are deemed to be at increased risk with pulmonary resection.

A number of factors need to be considered when selecting the ideal resection for a patient with a small peripheral NSCLC. One argument for performing the smallest resection possible that still allows complete removal of the cancer is that by sparing lung parenchyma, lung dysfunction, morbidity, and mortality are minimized. In an evaluation of 2200 lung resections, mortality was $6.2 \%$ after pneumonectomy, $2.9 \%$ after lobectomy, and $1.4 \%$ after lesser resections. ${ }^{12}$ These results suggest that perioperative mortality would be improved with a lesser resection. A positive finding that is often overlooked from the randomized LCSG study of SR and LR is the benefit from SR regarding preservation of pulmonary function. ${ }^{1}$ At 6 months the $\mathrm{FEV}_{1}$, forced vital capacity, and maximal voluntary ventilation were all significantly better in the SR group. At 12 months, the $\mathrm{FEV}_{1}$ was still significantly better. Other studies have also demonstrated better preservation of pulmonary function with SR. ${ }^{13,14}$

Higher local recurrence has been the major factor leading most North American surgeons to abandon SR, except for 


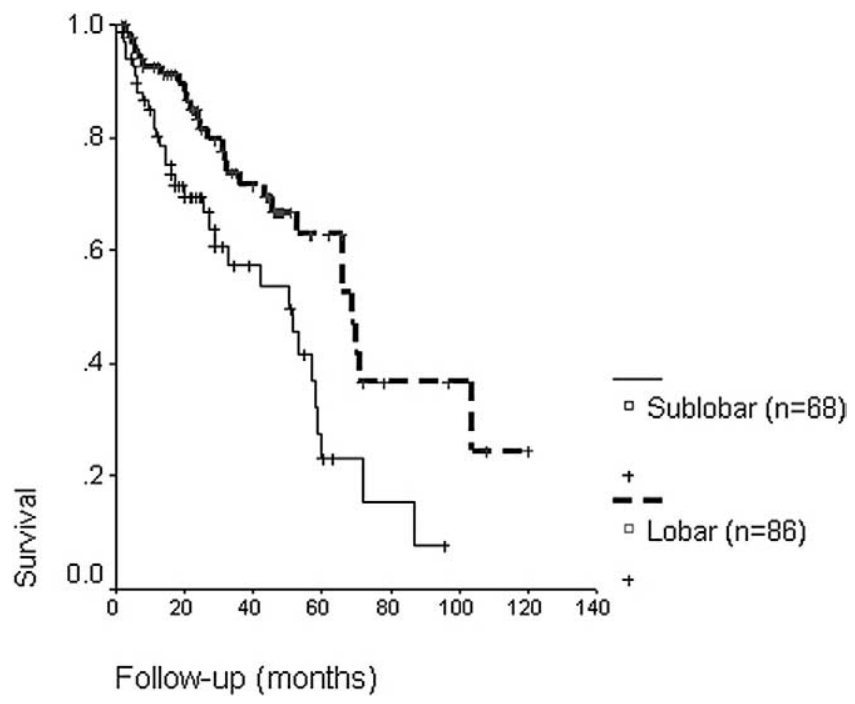

Figure 2. Survival for 2- to 3-cm tumors; SR versus $\operatorname{LR}(P=.003)$.

compromised patients. The Rush-Presbyterian group reported a $22 \%$ local recurrence rate for patients with stage I disease after SR, versus $4.9 \%$ after LR. ${ }^{15}$ Similarly, in the LCSG randomized trial, a 3-fold increase in local recurrence was seen with LR versus SR. ${ }^{1}$ Adjuvant external beam radiation is an option that has been reported to decrease local recurrence after wedge resection. ${ }^{16}$ In many ways, this approach is analogous to a combination of lumpectomy and external beam radiation, which has become standard therapy for many patients with anatomically favorable breast cancers. ${ }^{17}$ In the lung, however, difficulties in precisely delivering and developing a physics treatment plan for external beam radiation to the staple line may occur because of physiologic lung motion and the 3-dimensional irregularity of the target staple line after SR. SR and "postage stamp" external beam radiation therapy was recently investigated in a study coordinated by the Cancer and Leukemia Group. ${ }^{18}$ In their study, extended radiotherapy fields were often necessitated by the extent of the postoperative staple line, and the logistic concerns of daily radiotherapy placed a hardship on some patients. The advantage of brachytherapy is that it provides a means of delivering radiation in a more uniform manner, with $100 \%$ patient compliance and in the same setting as the lung resection. One study reported the use of brachytherapy in 33 high-risk patients, primarily after wedge resection. ${ }^{19}$ The local recurrence rate was $6.1 \%$, which was similar to the $6.4 \%$ reported after lobectomy in the LCSG study. ${ }^{1}$ A study from Pittsburgh compared local recurrence rates in 98 patients undergoing SR with brachytherapy and those in 102 patients undergoing SR alone. ${ }^{20}$ There was no difference in operative mortality, distal recurrence, or survival. However, local recurrence was significantly reduced, from $18.6 \%$ to $2 \%$, among those patients who received adjuvant brachytherapy. In our study, brachytherapy reduced local recurrence significantly to $3.3 \%$ among patients who underwent SR. Brachytherapy was also used for a higher proportion of the larger, 2- to 3-cm tumors, which may have accounted for the similarities in local recurrence rate between the LR and SR group patients with these larger tumors. The American College of Surgeons Oncology Group (ACOSOG) is currently developing a randomized study of adjuvant brachytherapy with SR versus SR alone for patients with T1 N0 NSCLC (Z4032). This study will include all NSCLC histologic types and will enroll patients who are considered to be at increased risk with LR. Cytologic samples will be obtained from the staple line in all cases as an additional evaluation of resection margin. It is to be hoped that the ACOSOG study will confirm the beneficial results of this and other nonrandomized reports regarding the use of adjunctive intraoperative brachytherapy with SR for NSCLC.

Tumor size is an important factor when planning intervention for NSCLC. One study from Japan reported on segmentectomy in 74 patients with T1 NO NSCLC. ${ }^{21}$ Overall 5-year survival was $82 \%$, but it was greater at $92 \%$ for tumors $2 \mathrm{~cm}$ or smaller than for larger tumors $(2.1-3 \mathrm{~cm})$, for which survival was $63 \%$ at 5 years. Additionally locoregional recurrence was seen in only $1.9 \%$ of the patients with smaller tumors, versus $33.3 \%$ of those with the larger tumors. Another Japanese study compared the results of extended segmentectomy with those of lobectomy for patients with T1 N0 tumors $2 \mathrm{~cm}$ or smaller. ${ }^{22}$ The 5 -year survivals were $87.1 \%$ in the segmentectomy group and $87.7 \%$ in the lobectomy group.

We accept the current consensus that lobectomy is the current standard of care for the treatment of stage IA NSCLC. However, our findings and those of others ${ }^{21,22}$ are compelling in suggesting that SR with brachytherapy may provide adequate local therapy, at least for tumors smaller than $2 \mathrm{~cm}$, even for low-risk patients. There remain some caveats. If smaller tumors are treated with SR, there remains a risk that lymph node metastases may be missed and that adequate staging of hilar and mediastinal lymph nodes might not accompany the resection. This was demonstrated in a series of 100 patients with NSCLC $1 \mathrm{~cm}$ or smaller, in which there was a 5\% incidence of unsuspected N1 disease. ${ }^{23}$ For this reason, we recommend lymph node dissection or sampling whenever SR is performed.

We believe that there are differences in the type of SR and favor anatomic segmentectomy when possible. We were surprised to see similar recurrence rates with segmentectomy and wedge resection. Factors that may have accounted for this are the small size of many of the tumors, the routine use of lymph node dissection or sampling, exclusion of patients with unsuspected nodal disease, and, finally, brachytherapy, which was used in $48 \%$ of the SR resections. 
Although many studies have demonstrated differences in local recurrence rates when comparing SR with LR, survivals are usually similar. In this study, no differences were seen in survival with the smaller $(<2 \mathrm{~cm})$ tumors, despite physiologic differences favoring the LR group. With the 2to 3-cm tumors, however, there was a marked difference in survival, favoring the lobectomy group. Our data (although not statistically significant) suggest that this difference may have been attributable to cancer-related death.

In conclusion, adjuvant brachytherapy appears to be a promising modality that may reduce local recurrence rate after SR. Additionally there are differences in outcome between smaller and larger T1 N0 cancers. Until further randomized studies are available, lobectomy should be used whenever possible. If the results of the upcoming ACOSOG study confirm the results from the preliminary studies on the use of brachytherapy, we suggest that a follow-up trial compare SR with brachytherapy versus lobectomy for all patients with small $(<2 \mathrm{~cm})$ NSCLC.

\section{References}

1. Ginsberg RJ, Rubinstein LV. Randomized trial of lobectomy versus limited resection for T1 N0 non-small cell lung cancer. Ann Thorac Surg. 1995;60:615-23.

2. Landreneau RJ, Sugarbaker DJ, Mack MJ et al. Wedge resection versus lobectomy for stage I (T1 N0 M0) Non-small cell lung cancer. J Thorac Cardiovasc Surg. 1997;113:691-700.

3. Koike T, Yamato Y, Yoshiya K, Kina S, Shimoyama T, Suzuki R, et al. Criteria for intentional limited pulmonary resection in cT1 N0 M0 peripheral lung cancer. Jpn J Thorac Cardiovasc Surg. 2003;51:515-9.

4. Henschke CI, McCauley DI, Yankelevitz DF, Naidich DP, McGuinness G, Miettinen OS, et al. Early Lung Cancer Action Project: overall design and findings from baseline screening. Lancet. 1999;354:99-105.

5. Yoshikawa K, Tsubuto N, Kodama K, Ayabe H, Taki T, Mori T. Prospective study of extended segmentectomy for small lung tumors: the final report. Ann Thorac Surg. 2002;73:1055-9.

6. Okada M, Sakamoto T, Nishio W, Uchino K, Tsubota N. Characteristics and prognosis of patients after resection of non-small cell lung carcinoma measuring $2 \mathrm{~cm}$ or less in greatest dimension. Cancer. 2003;98:535-41.

7. D'Amato TA, Galloway M, Szydlowski G, Chen A, Landreneau RJ. Intraoperative brachytherapy following thoracoscopic wedge resection of stage I lung cancer. Chest. 1998;114:1112-5.

8. Kupelian PA, Komaki R, Allen P. Prognostic factors in the treatment of node-negative non-small cell lung carcinoma with radiotherapy alone. Int J Radiat Oncol Biol Phys. 1996;36;607-13.

9. Zierhut D, Bettscheider C, Schubert K, van Kampen M, Wannenmacher M. Radiation therapy of stage I and II non-small cell lung cancer (NSCLC). Lung Cancer. 2001;34 Suppl 3:S39-43.

10. Sibley GS, Jamieson TA, Marks LB, Anscher MS, Prosnitz LR. Radiotherapy alone for medically inoperable stage I non-small cell lung cancer: the Duke experience. Int J Radiat Oncol Biol Phys. 1998;40:149-54.

11. Herrera LJ, Fernando HC, Perry Y, Gooding WE, Buenaventura PO, Christie NA, et al. Radiofrequency ablation of pulmonary malignant tumors in nonsurgical candidates. J Thorac Cardiovasc Surg. 2003; 125:929-37.

12. Ginsberg RJ, Hill LD, Eagan RT, Thomas P, Mountain CF, Deslauriers J, et al. Modern thirty-day operative mortality for surgical resections in lung cancer. J Thorac Cardiovasc Surg. 1983;86:654-8.

13. Takizawa T, Haga M, Yagi N, Terashima M, Uehera H, Yokoyama A, et al. Pulmonary function after segmentectomy for small peripheral carcinoma of the lung. J Thorac Cardiovasc Surg. 1999;118:536-41.
14. Keenan RJ, Landreneau RJ, Maley RH Jr, Singh D, Macherey R, Bartley S, et al. Segmental resection spares pulmonary function in patients with stage I lung cancer. Ann Thorac Surg. 2004;78:228-33.

15. Warren WH, Faber LP. Segmentectomy versus lobectomy in patients with stage I pulmonary carcinoma. J Thorac Cardiovasc Surg. 1994; 107:1087-94.

16. Miller JI, Hatcher CR. Limited resection of bronchogenic carcinoma in the patient with marked impairment of pulmonary function. Ann Thorac Surg. 1987;44:340-3.

17. Kini VR, White JR, Horwitz EM, Dmuchowski CF, Martinez AA, Vicini FA. Long term results with breast-conserving therapy for patients with early stage breast carcinoma in a community hospital setting. Cancer. 1998;82:127-33.

18. Shennib H, Bogart JA, Herndon J, Kohan L, Sugarbaker DJ, Keenan $\mathrm{RJ}$, et al. Thoracoscopic wedge resection and radiotherapy for T1 N0 non-small cell lung cancer (NSCLC) in high risk patients: preliminary analysis of a Cancer and Leukemia Group B and Eastern Cooperative Oncology Group phase II trial [abstract 240]. Int J Radiat Oncol Bio Phys. 2000;48(Suppl):232.

19. Lee W, Daly BD, DiPetrillo TA, Morelli DM, Neuschatz AC, Morr J, et al. Limited resection for non-small cell lung cancer: observed local control with implantation of I-125 brachytherapy seeds. Ann Thorac Surg. 2003;75:237-42.

20. Santos R, Colonias A, Parda D, Trombetta M, Maley RH, Macherey R, et al. Comparison between sublobar resection and ${ }^{125} \mathrm{I}$ brachytherapy after sublobar resection in high-risk patients with stage I non-smallcell lung cancer. Surgery. 2003;134:691-7.

21. Bando T, Yamagihara K, Ohtake Y, Miyahara R, Tanaka F, Hasegawa $\mathrm{S}$, et al. A new method of segmental resection for primary lung cancer: intermediate results. Eur J Cardiothorac Surg. 2002;21:894-9.

22. Okada M, Yoshikawa K, Hatta T, Tsubota N. Is segmentectomy with lymph node assessment an alternative to lobectomy for non-small cell lung cancer of $2 \mathrm{~cm}$ or smaller? Ann Thorac Surg. 2001;71:956-61.

23. Miller DL, Rowland CM, Deschamps C, Allen MS, Trastek VF, Pairolero PC. Surgical treatment of non-small cell lung cancer $1 \mathrm{~cm}$ or less in diameter. Ann Thorac Surg. 2002;73:1545-50.

\section{Discussion}

Dr Joseph P. Shrager (Philadelphia, Pa). It's a pleasure to be able to discuss one of your articles. I congratulate you and your colleagues on this potentially very important work and on the foresight of starting to use brachytherapy all the way back before 1997, as I gather.

Although the question of the equivalence of SR and lobectomy had seemingly been put to rest by the LCSG trial, there has been increasing interest in this subject as we have begun to detect more small tumors. The interest in these procedures has, appropriately I think, been heightened by the additional evidence that small tumors that are predominantly bronchoalveolar cell cancer (BAC) have a very low likelihood of nodal metastasis and can be reliably identified by preoperative computed tomographic scan criteria.

Several retrospective studies, including the one you nicely presented here today, have been interpreted as suggesting that either all tumors below a certain size, or at least small BACpredominant tumors, can be effectively managed with SR. However, I believe it's appropriate to sound a note of caution on this issue. Although I applaud the carefulness with which your conclusions are offered, it's critical to reiterate that any number of retrospective studies cannot compete with the scientific validity of a single large, prospective, randomized trial, such as the LCSG trial. Further, the initial rationale for SR-that many patients cannot tolerate a lobectomy-has always struck me as somewhat weak, and I think it only gets weaker with time as we increasingly 
recognize that patients with emphysema tend to have a "volume reduction benefit" from their lobectomy.

Finally, other than BAC tumors, there is clear evidence from the Mayo and from Japan that as many as $30 \%$ of patients with tumors smaller than $2 \mathrm{~cm}$ have $\mathrm{N} 1$ or N2 disease.

Looking specifically at the data you presented here, I believe that they also do not support a conclusion that SR alone without brachytherapy is broadly reasonable even for lesions smaller than $2 \mathrm{~cm}$. In the absence of brachytherapy, your reported local recurrence rate in this group was about $26 \%$. With more patients and a longer follow-up, one would have to believe that this would translate into a survival difference. On the other hand, I believe the data that you present here and that others have presented are very impressive with regard to the effectiveness of brachytherapy in this setting. I think the data on this issue strongly suggest that for the rare patient who is truly not a candidate for a lobectomy, adding brachytherapy to SR may well be appropriate. And if we ever do cross that threshold of sufficient evidence proving that SR is equivalent to lobectomy for some subset of noncompromised patients, then I believe it's likely that we will be doing that in conjunction with brachytherapy, on the basis of what you show here and what others have shown.

I agree with you that this is an area ripe for a planned, prospective, clinical trial, and ultimately it's quite possible that this approach will be proved to be appropriate for some subset of tumors. Until definitive data become available, however, I feel pretty strongly that it's the responsibility of this specialty, of the people in this room, to strongly advocate lobectomy unless a patient is considered to be at extraordinary risk with this operation. I think the temptation to do an easier operation, such as a wedge resection, is so great that if the thought leaders in this area do not continue to advocate lobectomy unless there are very strong data to the contrary, we will have potentially inappropriate wedge resections being done as common practice.

I have the following specific questions. First, you did not tell us here today and I don't see in the article exactly how the margins were checked intraoperatively. Everyone in this room knows the pitfalls of trying to get frozen sections on stapled margins. I have been impressed with the work from a couple of Japanese groups demonstrating that two methods of checking cytology on stapled margins are highly accurate in predicting local recurrence or lack of local recurrence.

Dr Patterson. Would you answer the question about the frozen section?

Dr Fernando. In terms of the intraoperative management, this is a difficult thing to answer in this retrospective multicenter study. I think that the future ACOSOG study will better address this, because we plan to look at the margins and we also plan to do a smear of the staple line as part of that protocol. I think this is an important issue, and this may be where brachytherapy has the most importance in that you do get a better margin.

When you do wedge resection at the moment, you are usually doing this for a compromised patient, and I think you are going to find patients with margins that are close. The addition of brachytherapy may improve this, which may account for the local recurrence rates we are seeing. Also, in the Japanese data, as you know, they do very extensive lymph node dissections and maybe in some cases extended segmentectomies, and they still seem to have these low local recurrence rates which we don't see over here in the United States with limited resection.

Dr Shrager. You didn't tell us the histologic types of your tumors. I would think that BACs are likely to be the subset of tumors for which we would ultimately be doing this sort of procedure. So what were the percentage of tumors that were pure or predominantly BAC?

Dr Fernando. I do not have that data at this time. However, it was a small percentage.

Dr Shrager. If the concept of a wedge resection for small tumors begins to gain acceptance, I think the natural course of events will be that a lot of people will be doing that by videoassisted thoracoscopic surgery. My personal belief is that this would lead to more incomplete resections and inferior lymph node dissections. Do you know what percentage of these were videoassisted thoracoscopic surgery versus open wedge resections?

Dr Fernando. It was a very small percentage, I think about $5 \%$. I do not know the answer exactly, but it was a small percentage.

Dr Thomas A. D'Amico (Durham, NC). I have two questions. How do you explain the apparent paradox of the higher local recurrence rate for smaller tumors than for larger tumors?

Dr Fernando. I wondered about that, and I believe that this may have been due to the greater use of brachytherapy for these larger tumors. On the other hand, you may expect closer margins with the larger tumors, so I cannot explain these results from our current data.

Dr D'Amico. Second, maybe you could share your thinking regarding for which patients you would do SR, because I think that probably matters. For example, you would never do a posterior segmentectomy on someone with advanced emphysema, leaving the worst anterior and apical segments in. You would do a lobectomy. For someone with restrictive lung disease and a superior segment tumor, you probably would perform segmentectomy. Does that go into your thinking when you plan SR versus LR?

Dr Fernando. I think, first of all, these have to be peripheral, not central tumors. At the current time, we only use SR for those patients whom we consider to be compromised and believe would benefit from that lesser resection. I think if you have a tumor nodule within the upper lobe in an area of the worst emphysema, it makes more sense to do a lobectomy with the current data. As we develop the long-term data from the ACOSOG study, I think we really have to look at what we should be doing for these smaller tumors.

Dr William H. Warren (Chicago, Ill). Your methods lead me to conclude that you think your local recurrences are only in your staple line. As someone who has gone on record critical of segmentectomy, I'm sure I've seen drop metastases and recurrence from nodes that are deep in the lobe and not resected with the segment. Can you please define for us what you mean by local recurrence?

Dr Fernando. Local recurrence is within the same tumor lobe and also includes hilar lymph nodes as well.

Dr Warren. That would not be treated with the brachytherapy?

Dr Fernando. The lymph nodes would not be treated by the brachytherapy.

Dr Joseph Miller (Atlanta, $G a$ ). I have two quick points. One regards your classification of poor pulmonary function. The $\mathrm{FEV}_{1}$ was listed as $53 \%$ in your abstract. I would submit that this is not poor pulmonary function. You could probably easily get away with 
a lobectomy in that group. Also, just briefly, I offer a word a caution. I do not think we need to move forward into this. In the 1980 s, we published a group with pulmonary function of $35 \%$ to $40 \%$ comparing 20 versus 20 treated with cone-down radiation at that point to 4500 rads. The recurrence rate went from $20 \%$ without radiation to $7.5 \%$, which is close to yours. But I do not think we want to move toward doing this operation with brachytherapy if, when the patients have an $\mathrm{FEV}_{1}$ of $51 \%$, a lobectomy can be done, which has remained the criterion standard.

Dr Fernando. I disagree a little bit, Dr Miller. We do quote your article in our manuscript. External beam radiation was also looked at by the Cancer and Leukemia Group in a study with postage stamp radiation after sublobar resection. The problem with external beam radiation is that it is difficult to precisely identify the staple line and there is the additional issue of lung motion. The advantage of brachytherapy is that you are placing this right over the staple line, delivering the radiation in a more uniform fashion, and minimizing radiation injury to the remaining lung.
I agree that you could perform a lobectomy on many of these patients, but you have to also look at the quality of life. If you look at the Lung Cancer Study Group data, at 6 months in three pulmonary function parameters, pulmonary function was actually better in the segmentectomy group. At 12 months the FEV was still better in the segmentectomy group. I think if you preserve or improve quality of life, you achieve the same survival and also decrease the local recurrence rate to the same as lobectomy, then I believe you are doing the patients a service.

Dr Thomas M. Egan (Chapel Hill, NC). Because any amount of radiation damages normal lung, do you have postoperative pulmonary function test results and diffusing capacity for carbon monoxide values for any of these patients?

Dr Fernando. We do not in this particular study. Again, that is going to be looked at in the ACOSOG study; the plan is to look at postoperative pulmonary function and also to look at dyspnea scores in these patients as well.

\section{JTCVS On-Line Manuscript Submission and Review} Please visit http://www.editorialmanager.com/jtcvs/

Effective September 15, 2001, authors and reviewers may submit manuscripts and reviews electronically via Editorial Manager, our new Web-based system with full electronic submission, review, and status update capabilities.

As we move from paper to electronic submissions, the Editorial Office will make proxy submissions of all manuscripts accompanied by a diskette containing the electronic files of the text, tables, and figures. Editors, authors, and reviewers will receive automatic e-mails when significant events occur.

We strongly encourage all authors and reviewers to use Editorial Manager. Although we will continue to accommodate the submission of paper manuscripts for some months, our goal is to be completely electronic within 9 to 12 months.

All individuals currently in our database for whom we have e-mail addresses will receive via e-mail a system-assigned username and password that can be used to log in to the system without prior registration. All those not receiving the e-mail must register the first time they use the system.

As with any broad systemic change, the conversion to the new system will take some time to complete. We ask your patience as we replace our in-office database with the new system. We also encourage you to take advantage of the speed and efficiency that the new system will provide for us all: editor, author, reviewer, and publisher. 